\title{
TITLE: PATTERNS OF PRACTICE SURVEY FOR BRACHYTHERAPY FOR CERVIX CANCER IN AUSTRALIA AND NEW ZEALAND
}

Running title: Brachytherapy survey for cervix cancer

\author{
Authors: \\ Karen $\operatorname{Lim}^{1,2}$ \\ Sylvia Van Dyk \\ Pearly Khaw ${ }^{4}$ \\ Jacqueline Veera ${ }^{1,2}$ \\ Linda Mileshkin ${ }^{4}$ \\ Lucy Ohanessian $^{1}$ \\ Michelle Harrison ${ }^{1,4}$ \\ Shalini K. Vinod ${ }^{1,2,3}$
}

1. Cancer Therapy Centre, Liverpool Hospital, Corner Campbell \& Goulbourn St, Liverpool, NSW2170

2. South Western Sydney Clinical School, UNSW, Medical Education and Research Precinct Level 2, Clinical Building, Liverpool Hospital, Cnr Elizabeth and Goulburn Sts, LIVERPOOL, NSW 2170

3. Western Sydney University, , Medical Education and Research Precinct

Level 2, Clinical Building, Liverpool Hospital, Cnr Elizabeth and Goulburn Sts, LIVERPOOL, NSW 2170

4. Peter MacCallum Cancer Centre, $\mathbf{3 0 5}$ Grattan Street, Parkville, Victoria $\mathbf{3 0 0 0}$

5. Chris O'Brien Lifehouse, Royal Prince Alfred Hospital, Missenden Road, Camperdown,

NSW 2050

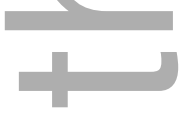

\section{Corresponding author:}

Dr Karen Lim

Cancer Therapy Centre

Liverpool Hospital

Locked Bag 7103

Liverpool BC, NSW 1871,

This is the author manuscript accepted for publication and has undergone full peer review but has not been through the copyediting, typesetting, pagination and proofreading process, which may lead to differences between this version and the Version of Record. Please cite this article as doi: 10.1111/1754-9485.12614

This article is protected by copyright. All rights reserved 
Australia.

Phone: +612 87389805

Fax: +612 87389819

Email: Karen.Lim@sswahs.nsw.gov.au

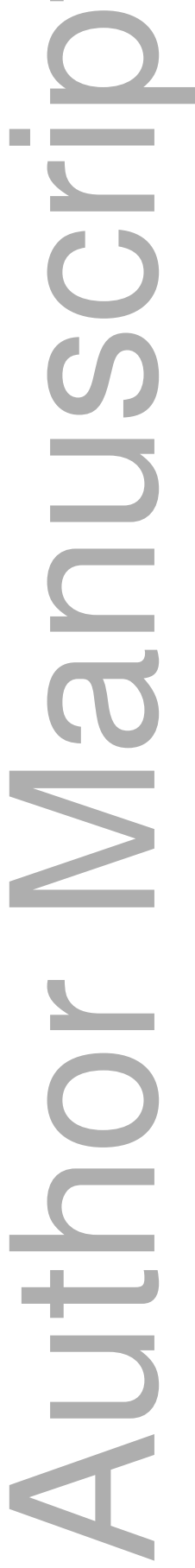

This article is protected by copyright. All rights reserved 
2 DR. KAREN LIM (Orcid ID : 0000-0002-4861-2108)

3 A/PROF. SHALINI KAVITA VINOD (Orcid ID : 0000-0001-8075-6219)

4

5

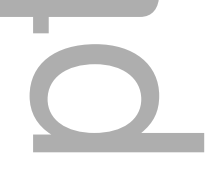

6 Received Date : 30-Nov-2016

7 Revised Date : 22-Feb-2017

8 Accepted Date: 11-Mar-2017

9 Article type : Radiation Oncology Original Article

10

11

12

ABSTRACT

13 Introduction

14 The purpose of this survey was to explore the current patterns of practice for brachytherapy

15 in cervix cancer in Australia and New Zealand. The survey was also intended to explore

16 clinician attitudes toward image-guided adaptive brachytherapy (IGABT) and identify barriers

17 to the implementation of IGABT

18 Methods

19 Electronic surveys were sent to all radiotherapy centres in Australia and New Zealand under

20 collaboration with Australia New Zealand Gynaecology and Oncology Group (ANZGOG), in

21 order to identify patterns of radiotherapy practice. The survey was sent out in December

22 2013, with a reminder in February 2014.

23 Results

24 Of the 75 radiotherapy centres in Australia and New Zealand, 23 centres replied ( $31 \%$

25 response rate). Twenty two responding departments treat cervix cancer with external beam

This article is protected by copyright. All rights reserved 
radiation (EBRT) (22/23; 96\%). Fourteen responses were from departments that also use intracavitary brachytherapy $(14 / 22 ; 64 \%)$. The remaining eight departments who do not offer intracavitary brachytherapy referred their patients on to other centres for brachytherapy. Ultrasound was used by $86 \%$ for applicator guidance. CT and MRI were used by $79 \%$, and $50 \%$ respectively for planning. Optimisation was based on organs at risk (93\%) and target volumes 31 (64\%).

\section{Conclusions}

Brachytherapy remains an integral component of definitive treatment for cervix cancer in

34 Australia and New Zealand. There was increased use of soft tissue imaging modalities with emphasis on verification; high rates of volumetric planning, and adherence to a defined overall treatment period. Brachytherapy was not substituted with other EBRT modalities. Despite this there remain barriers to implementation of image guided brachytherapy.

\section{Five key words:}

\section{Introduction}

Brachytherapy plays an integral role in the curative treatment of inoperable cervix cancer, enabling tumouricidal doses of radiation to be delivered directly to the primary site of disease $^{1}$. Recent SEER data suggests that brachytherapy utilisation in cervix cancer has declined in the USA from 83\% in 1988, to 58\% in 2009, with a sharp decline in $2003(43 \%)^{2}$. This decline in brachytherapy usage has been shown to impact adversely on cause-specific and overall survival ${ }^{2}$.

52 Brachytherapy has become more complex with advances in technology such as stepping source capabilities and the use of 3D imaging. It also requires more time and resources when

54 delivering fractionated courses of treatment. The advent of image guided adaptive brachytherapy (IGABT) over the last decade has resulted in a paradigm shift in the treatment

56 approach to cervix cancer from point based dosimetry to volume based dosimetry. This has

57 resulted in significant improvements to local control and reduced toxicity, Table $1^{3-16}$. 
58 Previous reports have demonstrated that centres in Australia are adopting image guided 59 protocols with an increase in the use of 3D imaging in brachytherapy planning from $27 \%$ in 602005 to $65 \%$ in $2009^{17,18}$. The purpose of this survey was to explore the current patterns of

61 practice for brachytherapy in cervix cancer in Australia and New Zealand. The survey was also 62 intended to explore clinician attitudes toward IGABT and identify barriers to the

63 implementation of IGABT.

64

Methods

The survey was distributed electronically via email to all radiotherapy centres affiliated with the Royal Australian and New Zealand College of Radiologists (RANZCR) and Australian and New Zealand Gynaecology Oncology Group (ANZGOG) across Australia and New Zealand in December 2013 with a reminder in February 2014. Survey responses were collated and analysed using descriptive analysis.

Results

Overview

Responses were received from all states and territories of Australia and New Zealand except for the Northern Territory. There are 75 radiotherapy centres in Australia and New Zealand, 23 of which treat cervix cancer with brachytherapy. Overall, 23 departments replied to the survey $(23 / 75=31 \%$ response rate). Twenty two responding departments treat cervix cancer with external beam radiation (EBRT) $(22 / 23=96 \%)$. Fourteen responses were from departments that also treat intracavitary brachytherapy (14/22=64\% response rate). The remaining eight departments who do not offer intracavitary brachytherapy all referred their patients on to other centres who do provide brachytherapy services. None of the survey respondents reported substituting brachytherapy with intensity modulated radiotherapy (IMRT) or stereotactic body radiation therapy (SBRT). Radiotherapy related survey responses were supplied by radiation therapists (43\%), and radiation oncologists (57\%). 
Over $95 \%$ of survey respondents used 3D conformal radiotherapy for the external beam component of treatment at the time of the survey. More conformal modalities such as IMRT, tomotherapy, volumetric arc therapy (VMAT) are also used but it is not clear in what clinical setting these were used (e.g. nodal boost).

94 Correspondingly, EBRT was prescribed to International Commission on Radiation Units (ICRU) 95 report 50 reference point in $64 \%$ of responses and to the $95 \%$ of the planning target volume 96 (PTV) in $45 \%$ of responses ${ }^{19}$. It is likely that the proportion of departments using 97 IMRT/tomotherapy/VMAT has increased since then.

98 The most common EBRT doses in use are $45 \mathrm{~Gy}$ in 25 fractions and $50.4 \mathrm{~Gy}$ in 28 fractions.

\section{Brachytherapy}

Overview

102 All respondents use high dose rate afterloaders, with one department also using pulse dose rate. Half the respondents commence brachytherapy after EBRT is completed, and half during week four or five of EBRT. The majority of respondents aimed to complete treatment in less than eight weeks (71\%), with 14\% aiming for less than seven weeks and $14 \%$ aiming for less than nine weeks. Most departments aim to treat with four fractions of brachytherapy (57\%) although logistical considerations may cause treatment courses to be contracted, two departments treat with three fractions and one department treats with six fractions. Interfraction intervals range from one week, 43\%; 4-5 days, 21\%; 2-3 days, 43\%; and 24 hours (7\%).

110 One department also treats twice daily occasionally. The majority of applicator insertions

111 occur in an operating theatre, (64\%), with the remainder (36\%) occurring in a dedicated

112 brachytherapy suite. All respondents reported using general anaesthetic for applicator

113 insertion. Spinal anaesthesia was also widely used (57\%) and epidural anaesthesia less so

114 (7\%). The prescription dose range from 3.5 - 8.5 Gy per fraction. The most common dosages

115 are 6 and 7 Gy per fraction.

Workload

118 The majority (71\%) of respondents treat more than ten patients per year, $21 \%$ treat five to ten patients per year and one centre treats between $60-70$ patients annually, Figure 1 .

121 The number of radiation oncologists performing procedures per department, varies from one (14\%), two (57\%), three (21\%) or four (7\%). 
123 Brachytherapy is offered to patients with metastatic disease by $79 \%$ of respondents.

124 The most common scenarios where this would be offered are indicated in Figure 2. In the

125 category "Other", respondents indicated that brachytherapy is offered on a case by case basis,

126 depending on patient Eastern Co-operative Oncology Group performance status (ECOG),

127 severity of local symptoms or clinical response to chemotherapy. The dose and fractionation

128 used in these scenarios are mostly identical to those used for radical patients, although $18 \%$

129 would use an abbreviated fractionation schedule depending on the scenario.

130

131

Use of Imaging

132 All respondents use some form of imaging at the time of brachytherapy to aid in treatment

133 planning, although not all considered this image guided brachytherapy. The majority of

134 centres offering brachytherapy use 3D imaging, Figure 3. Image guided brachytherapy is

135 performed by $71 \%$ of respondents, with the remaining $29 \%$ of respondents indicating they

136 would like to implement image guided brachytherapy in the future. Computed tomography

137 (CT) is the most commonly used form of 3D imaging (79\%) with $50 \%$ of the respondents using

138 magnetic resonance imaging (MRI) and 29\% using ultrasound. Many of the respondents use

139 multi-modality imaging. Most planning imaging takes place in the radiotherapy department

140 (79\%). Ultrasound is used to guide applicator insertion by $86 \%$ of respondents. It is also used

141 to aid planning by $29 \%$. Twenty nine percent of respondents take intrafraction verification

142 images after patient transfers and prior to brachytherapy treatment. Ultrasound is used by

$14321 \%$ and $x$-ray by $14 \%$ for intrafraction verification. CT and MRI are not used for intrafraction

144 verification. Of the $71 \%$ of respondents who do not take intrafraction verification images,

145 there is considerable reliance on skin markings to indicate applicator position. Twenty one

146 percent of respondents who take intrafraction verification images adjust the applicator

147 position if it is unsatisfactory, $7 \%$ replan based on the updated applicator position and $7 \%$

148 reimage and replan with CT. Use of 3D imaging is employed by $50 \%$ of respondents for every

149 fraction of brachytherapy. Interfraction imaging is used for replanning by $57 \%$ respondents,

150 while $29 \%$ back project the original plan onto the new image set. The purpose of interfraction

151 imaging for those not replanning is to verify the applicator position, 36\%; verify the target

152 volume, 21\%; and verify the organs at risk (OAR), $29 \%$.

153

154

Applicators and fixation

This article is protected by copyright. All rights reserved 
155

156

157

158

172

173

The majority of respondents used intracavitary applicators, tandem and ovoids, 93\%; tandem and ring, 29\%; and tandem and cylinder, $86 \%$. Use of combined interstitial and intracavitary applicators is $29 \%$. A vaginal spatula (to move the rectum away) is used by $50 \%$ of respondents. Applicators are fixed in position by use of intravaginal packing by $93 \%$ of respondents. Perineal sutures are used by $43 \%$, a perineal bar by $7 \%$, and mesh underpants by 14\%. Two departments also use fabric tape around the applicator to secure it in position.

\section{Planning methods}

All departments using brachytherapy to treat cervix cancer use imaging at the time of brachytherapy to aid treatment planning. 3D imaging is used by $79 \%$ (CT) and $50 \%$ (MRI) of respondents, with many using dual modality imaging. Brachytherapy planning methods range from plans based on applicator geometry, $36 \%$; optimised plans based on OAR dose constraints, 93\%; to optimised plans based on high risk clinical target volume (HRCTV) coverage, $64 \%$. Various degrees of contouring are performed on the 3D image data sets. The gross target volume (GTV) is contoured by $21 \%$, intermediate risk clinical target volume (IRCTV) by $29 \%$, HRCTV $64 \%$, rectum $93 \%$, bladder $86 \%$, sigmoid $79 \%$, small bowel $29 \%$, while the vagina is not contoured by any respondents.

\section{Prescription and dosimetry}

Thirty six percent of respondents prescribe brachytherapy to Point A, with 1 respondent (7\%) prescribing to Point $\mathrm{M}^{20}$. The Groupe Européen de Curiethérapie and the European Society of Radiotherapy and Oncology (GEC-ESTRO) defined HRCTV is used by $43 \%$ while $14 \%$ of respondents prescribe to a self-determined target volume. One department that prescribes to the HRCTV stated they continue to report dose to Point A.

Planning constraints for the bladder range from 68-90 Gy in equivalent doses to $2 \mathrm{~Gy}$ fractionation (EQD2). Constraints for the rectum and sigmoid range from $64-75$ Gy and 54 $75 \mathrm{~Gy}$ respectively. Only one respondent indicated a vaginal mucosal constraint of $85 \mathrm{~Gy}$. Only one respondent reported using a rectal probe to measure the rectal dose during treatment and commented that treatment is corrected if the dose is hotter than expected.

\section{Moving toward and improving image guided brachytherapy}

Twenty nine percent of respondents would like to move to image guided brachytherapy. The greatest impediment to implementation and improvement of image guided brachytherapy is

This article is protected by copyright. All rights reserved 

lack of access to MRI, 79\%. The responses indicated that respondents using CT would like to improve image guided brachytherapy by accessing MRI. Similarly, respondents who use MRI would like greater access to it. Two respondents felt they had insufficient patient numbers to pursue the infrastructure needed for image guided brachytherapy. Other resource constraints include lack of access to anaesthetic and operating suite services, and lack of funds to finance training and equipment.

\section{Discussion}

The current survey results represent an update on the status of brachytherapy for cervix cancer throughout Australia and New Zealand. Important findings are the increased use of soft tissue imaging modalities, high rates of volumetric planning, and adherence to a defined overall treatment period. Most importantly, brachytherapy is prescribed for all patients regardless of the primary hospital's ability to deliver it. Patients undergoing radiotherapy in hospitals where brachytherapy is not available are referred to other institutions to receive brachytherapy. No departments in this survey reported using alternative boost modalities such as IMRT, SBRT or VMAT. This is a striking finding and is in stark contrast to recent data from the United States where Gill et al identified a $10.5 \%$ reduction in the use of brachytherapy for cervix cancer and a corresponding $10.3 \%$ increase in the use of IMRT or SBRT for boosting the tumour ${ }^{10}$. The use of an IMRT or SBRT boost was found to be associated with a significant increase in mortality risk.

\section{Imaging}

The use of 3D imaging has greatly expanded since the last survey conducted throughout Australia and New Zealand in 2009, Table $2^{17,18}$. The high levels of CT use are to be expected as the majority of departments (79\%) perform imaging in the radiotherapy departments using their own CT scanners. Use of MRI has increased from $15 \%$ to $50 \%$ in the intervening years. Only one department uses MRI exclusively. This department has an MRI scanner in the radiotherapy centre. Use and expansion of soft tissue imaging throughout Australia and New Zealand compares favourably to other parts of the world, with uptake exceeding most other countries and regions, Table $3^{17,21-27}$. The most notable increase in soft tissue imaging has been in the use of ultrasound to aid applicator insertion (86\%) and treatment planning (29\%). While many practitioners have reported on the utility of using ultrasound for difficult 
221

222

223

224

225

226

227

228

229

230

231

232

233

234

235

236

237

238

239

240

241

242

243

244

245

246

247

248

249

250

251

252

253

insertions Small et al recommend using ultrasound for all insertions as they felt uterine perforation was possible in any patient ${ }^{28,29}$.

The use of intra-fraction imaging was asked for the first time in this survey. Intra-fraction imaging refers to imaging taken after planning imaging (and multiple patient transfers) and prior to brachytherapy treatment. The importance of intra-fraction imaging in modern image based protocols was described by Anderson et $a l^{30}$. This group reimaged patients during a single insertion to ascertain intra-fraction changes to the position of OAR. The average time between planning MRI and pretreatment MRI was 4.75 hours (range 3.2 - 9.9 hours). During this time, the position of the OAR changed and dose constraint compliance reduced by $13.9 \%$. The survey identified $21 \%$ of practitioners use ultrasound to verify the applicator position prior to treatment. While ultrasound verification cannot fully assess the position of OAR through the 2D keyhole view, it can confirm the target applicator relationship and ensure that isodose coverage beyond the target volume is safe for surrounding tissues ${ }^{31}$. This simple verification can reduce and correct applicator shifts that have been shown to result in mean changes to the bladder and rectum of $5 \%$ and $6 \%$ per $m m$ for $D_{2 \mathrm{~cm} 3}$ and $D_{0.1 \mathrm{~cm} 3}$ respectively ${ }^{32}$. Use of imaging to guide applicator insertion and verify applicator position improves the technical quality of implants which in turn improves local control.

\section{Applicators}

Conventional intracavitary applicators are used by the majority of practitioners, although the survey identified use of combined intracavitary interstitial applicators by $29 \%$ of respondents. This is a moderate uptake compared to world uptake of $5-44 \%$, Table 1. A vaginal spatula (rectal retractor) was used by $50 \%$ of respondents. The importance of using a retractor has been clearly demonstrated. Stitt et al. showed that use of retractor increased the distance to the rectum by $4-14 \mathrm{~mm}$, reducing the dose by an average of $30 \%{ }^{33}$. Similarly, it has been shown that use of a retractor leads to lower rectal and sigmoid doses when compared with vaginal packing alone ${ }^{34}$.

\section{Planning}

In the 2009 survey four departments (20\%) based contouring, treatment volumes and dose prescriptions on GEC-ESTRO recommendations ${ }^{17}$. This survey has identified nine departments (64\%) contouring the HRCTV and $43 \%$ optimising plans to this volume. Only seven

This article is protected by copyright. All rights reserved 
254 departments use MRI. This means two departments contour the HRCTV based on CT alone.

255 There have been significant differences noted in the width of the cervix as identified on CT

256 and MRI. Cervix width on CT has been shown to be wider than on MRI and resulted in

257 statistically significant differences in the volume treated to the prescription dose ${ }^{35}$. Of

258 interest is that the two departments using CT alone do employ ultrasound to aid applicator

259 insertion. Van Dyk et al. have measured cervix dimensions on ultrasound and MRI and shown

260 good agreement between the two modalities ${ }^{36}$. There is potential for departments using CT

261 alone to extend the role for ultrasound to assist in more accurately identifying the cervix.

262 There was high uptake of OAR contouring for rectum, bladder and sigmoid, less so for small

263 bowel (29\%) while the vagina was not contoured by any respondents. Reporting doses

264 received by the vagina was not recommended in ICRU report $38^{37}$. The latest report aimed at

265 prescribing, recording, and reporting brachytherapy for cancer of the cervix, ICRU report 89,

266 does recommend reporting vaginal doses and details methods based on the imaging used for

267 planning

Doses

271 The majority of departments deliver brachytherapy over four fractions prescribing $6-7 \mathrm{~Gy}$

272 per fraction. Combined with average EBRT doses of $45 \mathrm{~Gy}$ the mean total dose prescribed is

$27381.6 \mathrm{~Gy}_{10} \mathrm{EQD} 2$, (range $73.2-85.6 \mathrm{~Gy}_{10}$ ). The range of doses are somewhat more

274 conservative compared to the doses used by departments listed in Table 1, where the mean

275 dose is $82.7 \mathrm{~Gy}_{10}$ (range $73.1-96.5 \mathrm{~Gy}_{10}$ ). It should be noted that the current survey asked for

276 prescription doses and Table 1 records mean doses received by the target volumes. Smaller

277 volumes of disease would receive higher doses. The greater use of combined

278 interstitial/intracavitary applicators by departments listed in Table 1 also contributes to higher

279 absorbed tumour doses. Dose reporting was not specifically queried although one

280 respondent stated that dose was prescribed to the HRCTV and dose to Point A was

281 documented. Interestingly, ICRU report 89 recommends reporting dose at Point A be

282 continued even by departments employing 3D imaging to plan treatment ${ }^{38}$. This enables

283 comparison of doses over different eras and across different levels of planning complexity.

Workload

This article is protected by copyright. All rights reserved 
285 The burden of cervix cancer is not high in Australia and New Zealand, largely due to well

286 subscribed screening programs and high standards of living. However, there is a small

287 consistent caseload, particularly in areas of lower socioeconomic status and a higher

288 immigrant population. Managing smaller numbers of patients presents unique problems.

289 These include, maintaining skills, accessing resources and infrastructure, offering support

290 services, and following recommendations. An American patterns of care study by Eifel et al.

291 found that smaller departments treating few patients were more likely to treat with EBRT

292 alone, prolong overall treatment time and deliver lower doses of radiation to Point $A^{39}$.

293 Fortunately, the results of the current survey do not seem to mimic the American experience.

294 All departments that did not offer brachytherapy do refer patients to another facility and all

295 respondent brachytherapy facilities have treatment guidelines in place. For departments who

296 do find their caseload diminishing it may be prudent and pragmatic to refer their patients to

297 larger facilities for treatment ${ }^{40}$.

\section{Barriers to IGABT}

The main barriers to implementation of IGABT were access to MRI, budgetary constraints, anaesthetics/theatre access and insufficient patient numbers. The most common reason cited for not implementing IGABT was insufficient patient numbers. All the centres that had no inclination to implement IGABT, did not provide a cervix brachytherapy service. Given the capital costs, staff training, logistical difficulties and learning curve associated with this technique, it is understandable that these centres send their patients elsewhere for their brachytherapy. Of the centres that provide an intrauterine cervix brachytherapy service, $71 \%$ (10/14) reported already performing IGABT. The remaining 4 centres indicated that they would like to implement IGABT. Access to MRI remains the main barrier to implementing or improving 3D image based planning. Respondents already using MRI indicated that they would like to expand use of MRI but access to it remains difficult. In Australia, only one simulation and one dosimetry episode is funded per course of brachytherapy. Despite this lack of funding for replanning $57 \%$ of respondents replan each fraction.

\section{Limitations}

314 Not every centre in Australia \& New Zealand is represented in this survey and due to the

315 voluntary nature of the survey questionnaire, response bias is unavoidable. Another

316 limitation of this survey was that patient outcomes were not explored. This is of particular 
317

318

319

interest in light of the toxicity, local control and survival outcomes that are now being reported by the EMBRACE and Retro-EMBRACE collaborations ${ }^{41}$. Details regarding the imaging protocols used for those centres which incorporated MRI into their brachytherapy workflow were also not explored.

Patterns of care surveys are an important way of monitoring progress in the treatment of cervix cancer with brachytherapy. They can act as a means to benchmark treatment protocols and also draw attention to emerging research, updated guidelines and recommendations, and implementation of clinical trial outcomes. By necessity the questions asked by the survey must change to reflect changes in these entities. It is hoped that such surveys continue to be conducted and embraced within the Australian and New Zealand brachytherapy community.

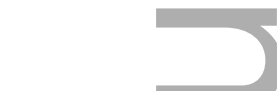

\section{Conclusions}

Brachytherapy remains an integral component of definitive treatment for cervix cancer in Australia and New Zealand. None of the survey respondents were willing to substitute it with IMRT or SBRT if brachytherapy was still technically possible.

Most of the survey respondents who offer brachytherapy to their cervix cancer patients demonstrate a substantial shift toward 3D IGABT techniques and volume based planning. There appears to be some heterogeneity in how brachytherapy dose is prescribed, reflecting the shift in approach from a purely geometrical applicator based prescription to one which takes into account individual anatomy.

Uptake of soft tissue imaging has increased significantly since 2009, with an emphasis on guiding applicator insertion and verifying applicator placement. Despite the high uptake of soft tissue imaging and volume based planning there are still perceived barriers to implementation of image guided brachytherapy.

This article is protected by copyright. All rights reserved 
References

1. Lanciano RM, Won M, Coia LR, et al. Pretreatment and treatment factors associated with improved outcome in squamous cell carcinoma of the uterine cervix: a final report of the 1973 and 1978 patterns of care studies. IntJRadiatOncolBiolPhys 1991;20:667676.

2. Han K, Milosevic M, Fyles A, et al. Trends in the Utilization of Brachytherapy in Cervical Cancer in the United States. International Journal of Radiation

Oncology*Biology*Physics 2013;87:111-119.

3. Pötter R, Georg P, Dimopoulos JCA, et al. Clinical outcome of protocol based image (MRI) guided adaptive brachytherapy combined with 3D conformal radiotherapy with or without chemotherapy in patients with locally advanced cervical cancer. Radiotherapy and Oncology 2011;100:116-123.

4. Charra-Brunaud C, Harter V, Delannes M, et al. Impact of 3D image-based PDR brachytherapy on outcome of patients treated for cervix carcinoma in France: Results of the French STIC prospective study. Radiotherapy and Oncology 2012;103:305-313.

5. Petit A, Floquet A, Lasbareilles $\mathrm{O}$, et al. Pulsed-dose-rate brachytherapy for uterine cervix carcinoma: 10 years of experience with 226 patients at a single institution. Brachytherapy 2013;12:542-549. dosimetric parameters of chemo-radiation including MRI guided adaptive brachytherapy with tandem-ovoid applicators for cervical cancer patients: A single institution experience. Radiotherapy and Oncology 2013;107:69-74.

7. Lindegaard JC, Fokdal LU, Nielsen SK, et al. MRI-guided adaptive radiotherapy in locally advanced cervical cancer from a Nordic perspective. Acta Oncologica 2013;52:15101519.

8. Rijkmans EC, Nout RA, Rutten IHHM, et al. Improved survival of patients with cervical cancer treated with image-guided brachytherapy compared with conventional brachytherapy. Gynecologic Oncology 2014;135:231-238. 9. Narayan K, van Dyk S, Bernshaw D, et al. Ultrasound guided conformal brachytherapy of cervix cancer: survival, patterns of failure, and late complications. Journal of Gynecologic Oncology 2014;25:8.

This article is protected by copyright. All rights reserved 
10. Gill BS, Kim H, Houser CJ, et al. MRI-Guided High-Dose-Rate Intracavitary Brachytherapy for Treatment of Cervical Cancer: The University of Pittsburgh Experience. International Journal of Radiation Oncology*Biology*Physics 2015;91:540-547.

11. Castelnau-Marchand P, Chargari C, Maroun P, et al. Clinical outcomes of definitive chemoradiation followed by intracavitary pulsed-dose rate image-guided adaptive brachytherapy in locally advanced cervical cancer. Gynecologic Oncology.

12. Choong ES, Bownes P, Musunuru HB, et al. Hybrid (CT/MRI based) vs. MRI only based image-guided brachytherapy in cervical cancer: Dosimetry comparisons and clinical outcome. Brachytherapy 2015.

13. Tharavichitkul E, Tippanya D, Jayavasti R, et al. Two-year results of transabdominal ultrasound-guided brachytherapy for cervical cancer. Brachytherapy $2015 ; 14: 238-244$

14. Ribeiro I, Janssen H, De Brabandere M, et al. Long term experience with 3D image guided brachytherapy and clinical outcome in cervical cancer patients. Radiother Oncol 2016;120:447-454.

15. Sturdza A, Potter R, Fokdal LU, et al. Image guided brachytherapy in locally advanced cervical cancer: Improved pelvic control and survival in RetroEMBRACE, a multicenter cohort study. Radiother Oncol 2016.

16. van Dyk S, Narayan K, Bernshaw D, et al. Clinical outcomes from an innovative protocol using serial ultrasound imaging and a single MR image to guide brachytherapy for locally advanced cervix cancer. Brachytherapy 2016.

17. van Dyk S, Byram D, Bernshaw D. Use of 3D imaging and awareness of GECESTRO recommendations for cervix cancer brachytherapy throughout Australia and New Zealand. J Medlmaging RadiatOncol 2010;54:383-387. van Dyk S, Byram D, Bernshaw D. Brachytherapy for cancer of the cervix: an Australian and New Zealand survey of current treatment techniques. J Med Imaging Radiat Oncol 2008;52:588-597.

19. ICRU. ICRU Report 50 Prescribing, Recording and Reporting Photon Beam Therapy In: TL, ed. Bethesda, Maryland International Commission on Radiation Units, 1993. 20. Thomadsen BR, Shahabi S, Stitt JA, et al. High dose rate intracavitary brachytherapy for carcinoma of the cervix: the Madison system: II. Procedural and physical considerations. IntJ RadiatOncolBiolPhys 1992;24:349-357.

This article is protected by copyright. All rights reserved 
21. Viswanathan AN, Erickson BA. Three-dimensional imaging in gynecologic brachytherapy: a survey of the American Brachytherapy Society. International Journal of Radiation Oncology Biology Physics 2010;76:104-109.

22. Guedea F, Venselaar J, Hoskin P, et al. Patterns of care for brachytherapy in Europe: Updated results. Radiotherapy and Oncology 2010;97:514-520.

23. Pavamani S, D'Souza DP, Portelance L, et al. Image-guided brachytherapy for cervical cancer: a Canadian Brachytherapy Group survey. Brachytherapy 2011;10:345-351.

24. Phan T, Mula-Hussain L, Pavamani S, et al. The changing landscape of brachytherapy for cervical cancer: a Canadian practice survey. Curr Oncol 2015;22:356-360. Tan LT. Implementation of image-guided brachytherapy for cervix cancer in the UK: progress update. ClinOncol(RCollRadiol) 2011;23:681-684. in Latin America: A patterns-of-care survey. Brachytherapy 2011;10:363-368.

27. Viswanathan AN, Creutzberg CL, Craighead P, et al. International brachytherapy practice patterns: a survey of the Gynecologic Cancer Intergroup (GCIG). IntJRadiatOncolBiolPhys 2012;82:250-255.

28. van Dyk S, Schneider M, Kondalsamy-Chennakesavan S, et al. Ultrasound use in gynecologic brachytherapy: Time to focus the beam. Brachytherapy 2015;14:390-400.

29. Small WJ, Strauss JB, Hwang CS, et al. Should Uterine Tandem Applicators Ever Be Placed Without Ultrasound Guidance? No: A Brief Report and Review of the Literature. International Journal of Gynecological Cancer 2011;21:941-944 910.1097/IGC.1090b1013e31821bca31853.

30. Anderson C, Lowe G, Wills R, et al. Critical structure movement in cervix brachytherapy. Radiotherapy and Oncology 2013;107:39-45. van Dyk S, Kondalsamy-Chennakesavan S, Schneider M, et al. Assessing changes to the brachytherapy target for cervical cancer using a single MRI and serial ultrasound. Brachytherapy 2015;14:889-897.

32. Tanderup K, Hellebust TP, Lang S, et al. Consequences of random and systematic reconstruction uncertainties in 3D image based brachytherapy in cervical cancer. RadiotherOncol 2008;89:156-163.

33. Stitt JA, Fowler JF, Thomadsen BR, et al. High dose rate intracavitary brachytherapy for carcinoma of the cervix: the Madison system: I. Clinical and radiobiological considerations. IntJ RadiatOncolBiolPhys 1992;24:335-348.

This article is protected by copyright. All rights reserved 
34. Gaudet M, Lim P, Yuen C, et al. Comparative analysis of rectal dose parameters in image-guided high-dose-rate brachytherapy for cervical cancer with and without a rectal retractor. Brachytherapy 2014;13:257-262.

35. Viswanathan AN, Dimopoulos J, Kirisits C, et al. Computed tomography versus magnetic resonance imaging-based contouring in cervical cancer brachytherapy: results of a prospective trial and preliminary guidelines for standardized contours. IntJ RadiatOncolBiolPhys 2007;68:491-498.

36. van Dyk S, Kondalsamy-Chennakesavan S, Schneider M, et al. Comparison of measurements of the uterus and cervix obtained by magnetic resonance and transabdominal ultrasound imaging to identify the brachytherapy target in patients with cervix cancer. Int $J$ Radiat Oncol Biol Phys 2014;88:860-865.

37. ICRU. Report 38 Dose and volume specification for reporting intracavitary therapy in gynecology Bethesda MD: International Commission of Radiation Units and Measurements, 1985.

38. ICRU. Report 89 Prescribing, recording, and reporting brachytherpay for cancer of the cervix. Bethesda, Maryland, 2013.

39. Eifel PJ, Moughan J, Erickson B, et al. Patterns of radiotherapy practice for patients with carcinoma of the uterine cervix: a patterns of care study. IntJRadiatOncolBiolPhys 2004;60:1144-1153.

40. Thompson SR, Delaney GP, Gabriel GS, et al. Patterns of care study of brachytherapy in New South Wales: cervical cancer treatment quality depends on caseload. Journal of Contemporary Brachytherapy 2014;6:28-32.

41. Tanderup K, Lindegaard JC, Kirisits C, et al. Image Guided Adaptive Brachytherapy in cervix cancer: A new paradigm changing clinical practice and outcome. Radiother Oncol 2016;120:365-369.

\section{Figure Legends}

Figure 1. Average number of patients with cervix cancer treated per department

Figure 2. Percentage of departments offering brachytherapy for metastatic disease

This article is protected by copyright. All rights reserved 
479 Figure 3. Percentage of departments using imaging

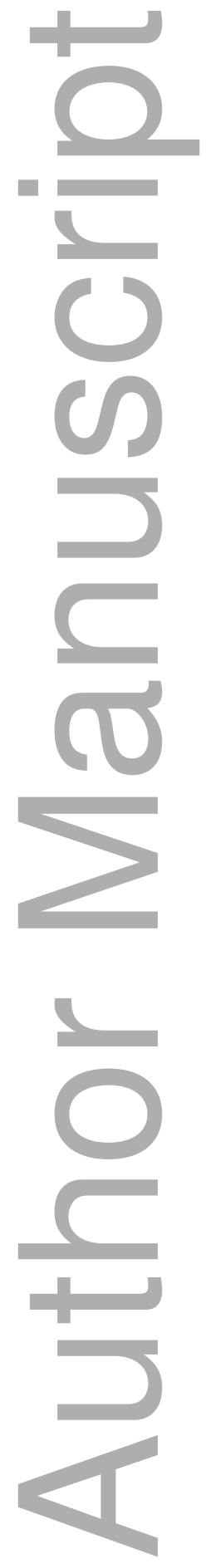

This article is protected by copyright. All rights reserved 
Table 1. Literature review of clinical outcomes from image based brachytherapy

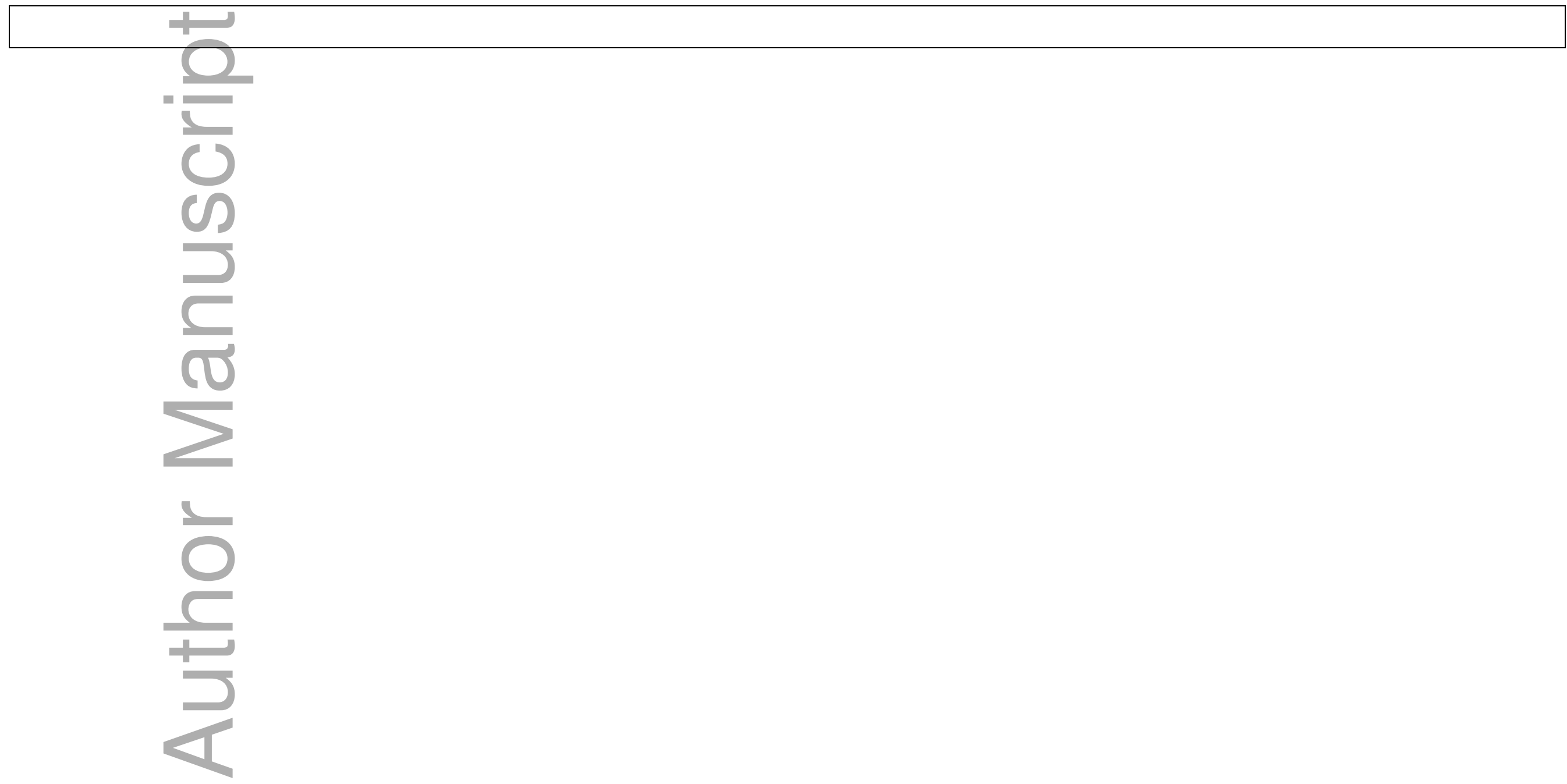

This article is protected by copyright. All rights reserved 
Table 2. Comparison of imaging modalities used throughout Australia and New Zealand over three survey periods

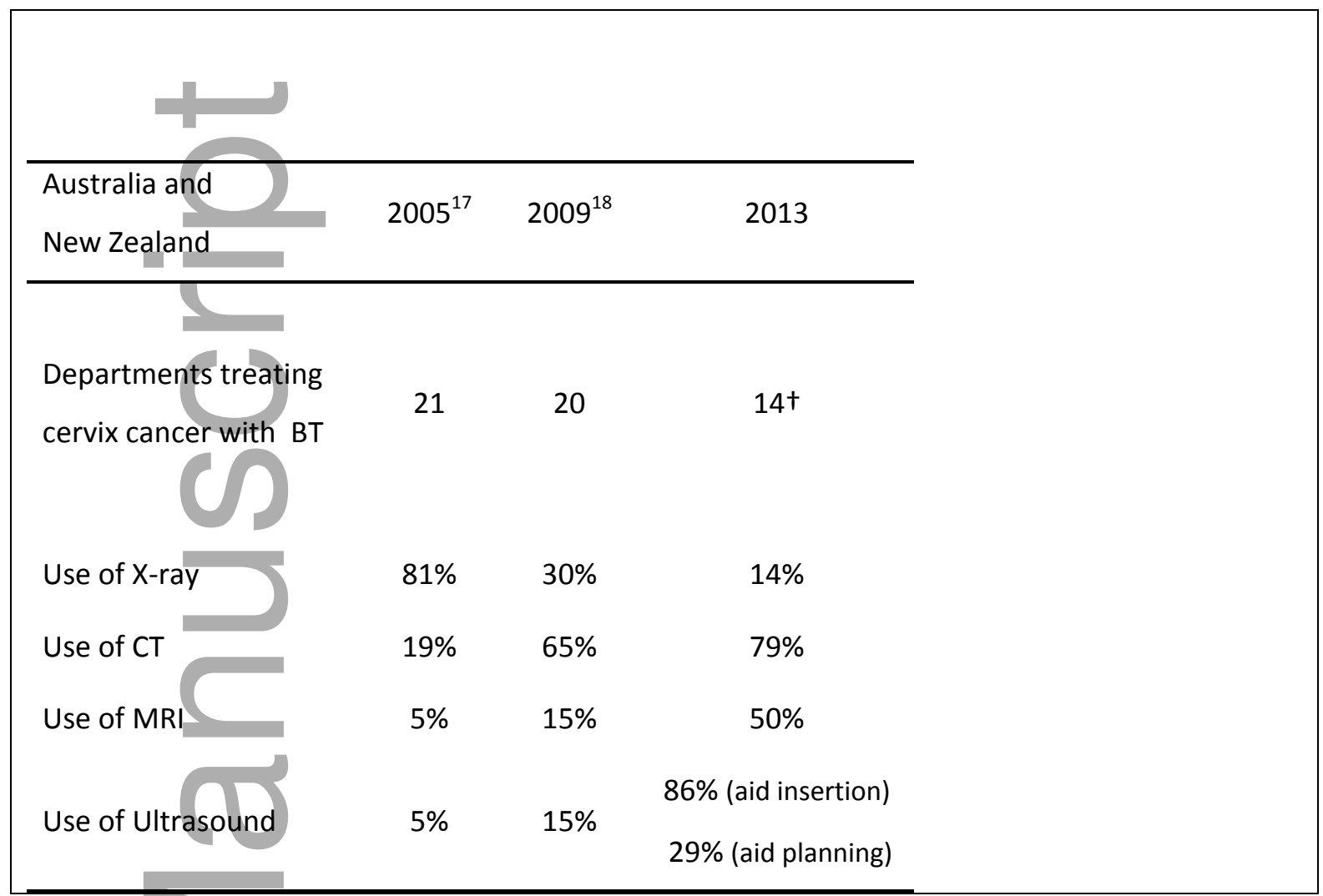

†14 respondents practice brachytherapy

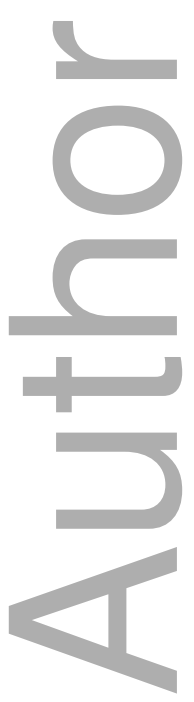

This article is protected by copyright. All rights reserved 
Table 3. Comparison of imaging modalities used for brachytherapy planning throughout the world

Imaging modality used for planning

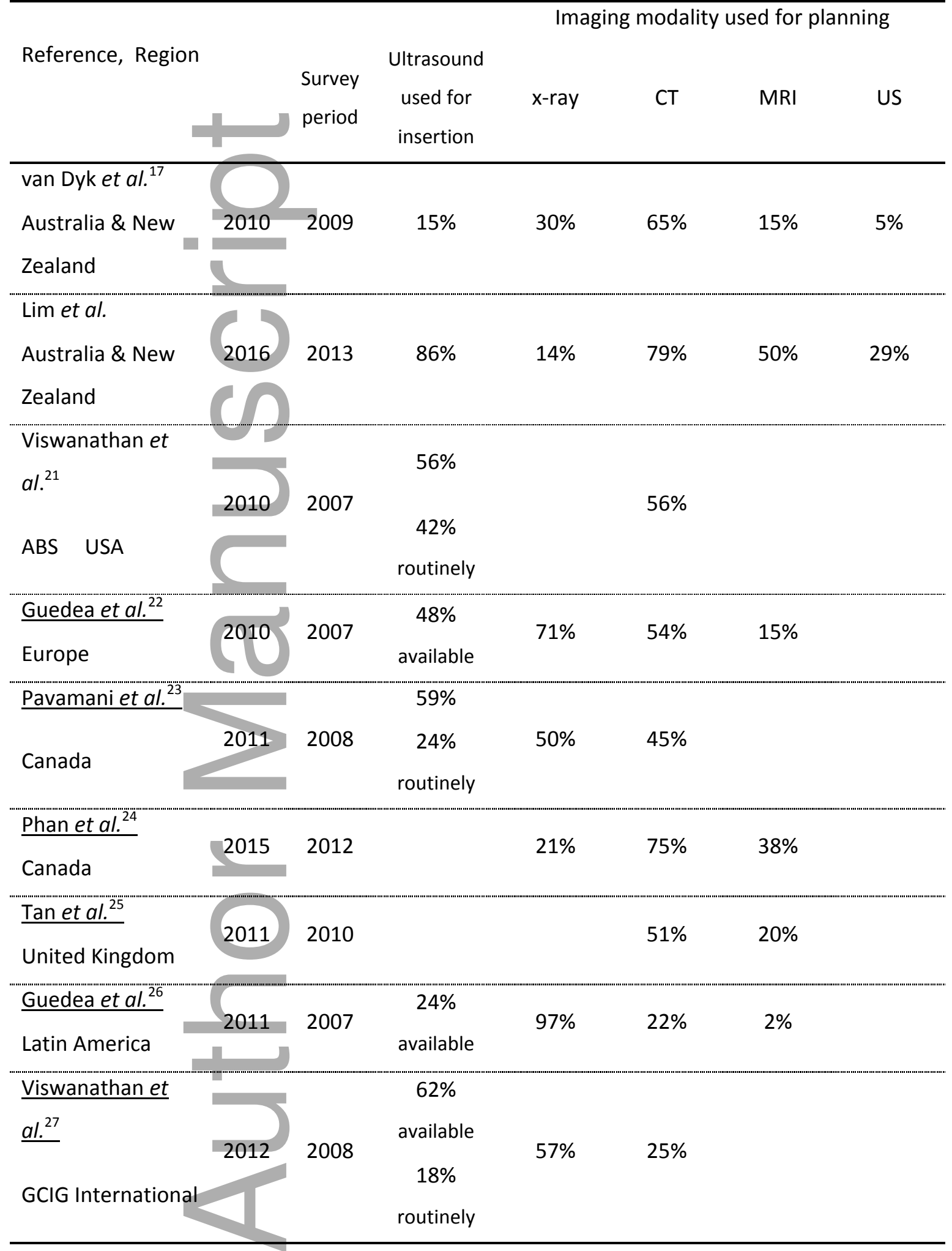

ABS American

Brachytherapy

Society; USA

United States of

America; GCIG

Gynecological

Cancer

Intergroup.

This article is protected by copyright. All rights reserved 


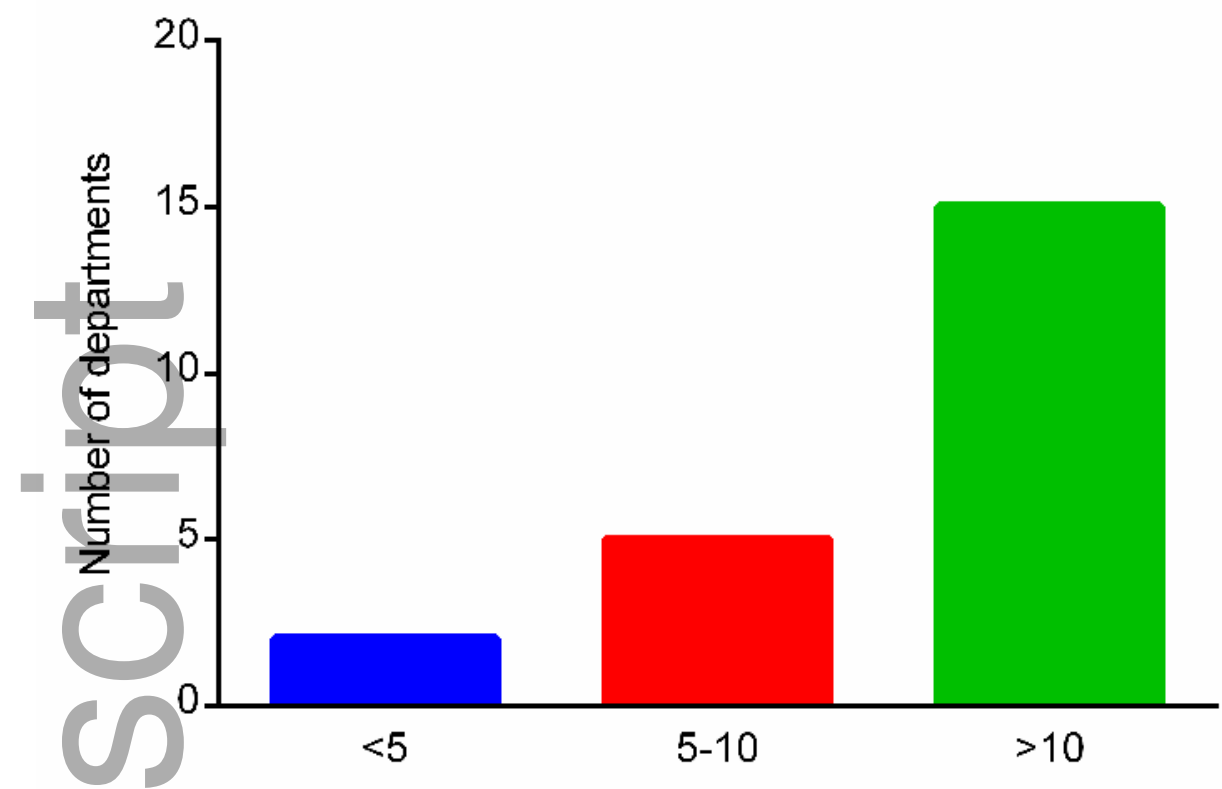

Average number of cervix patients treated per year

ara_12614_f1.tif 

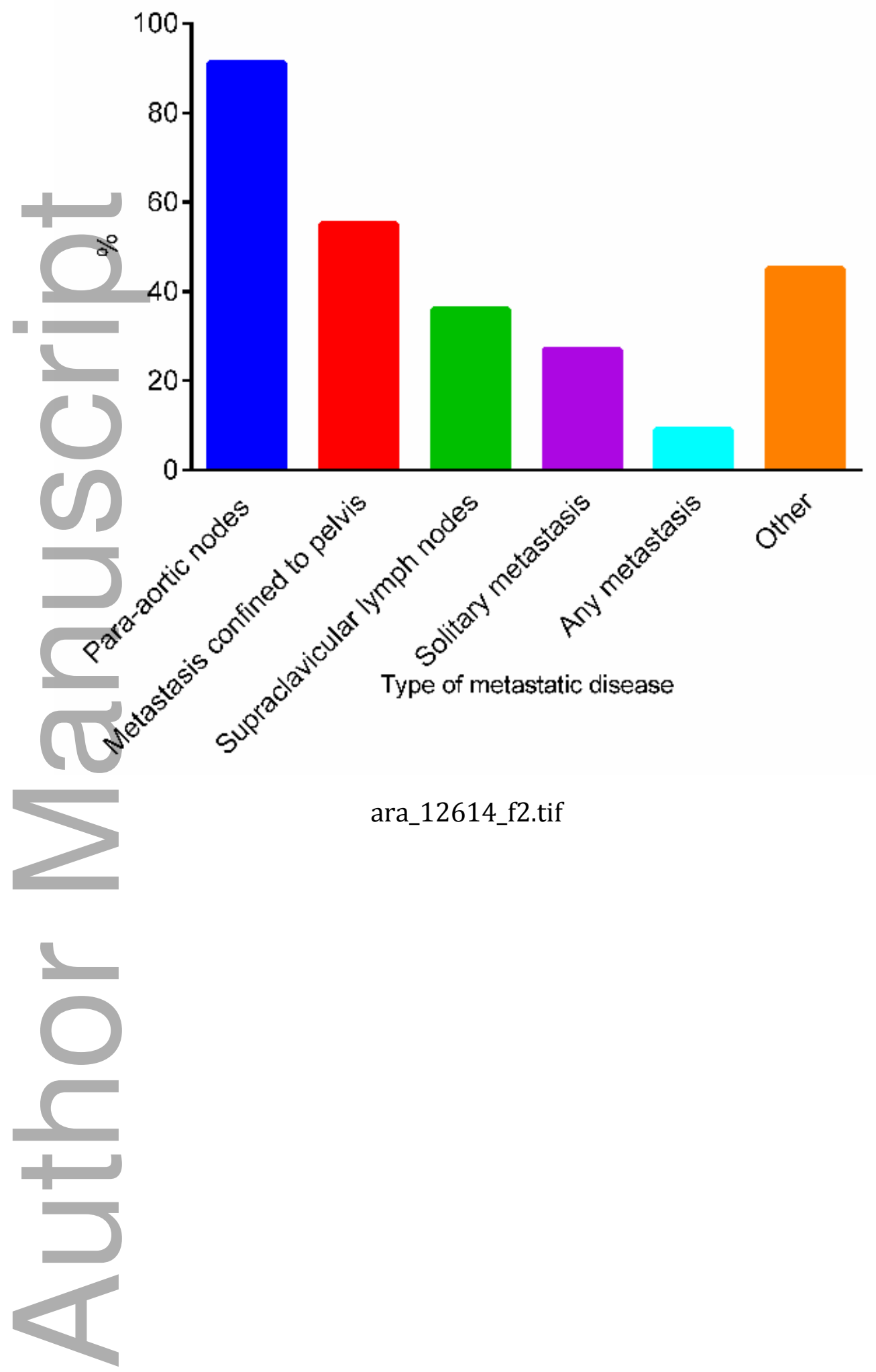


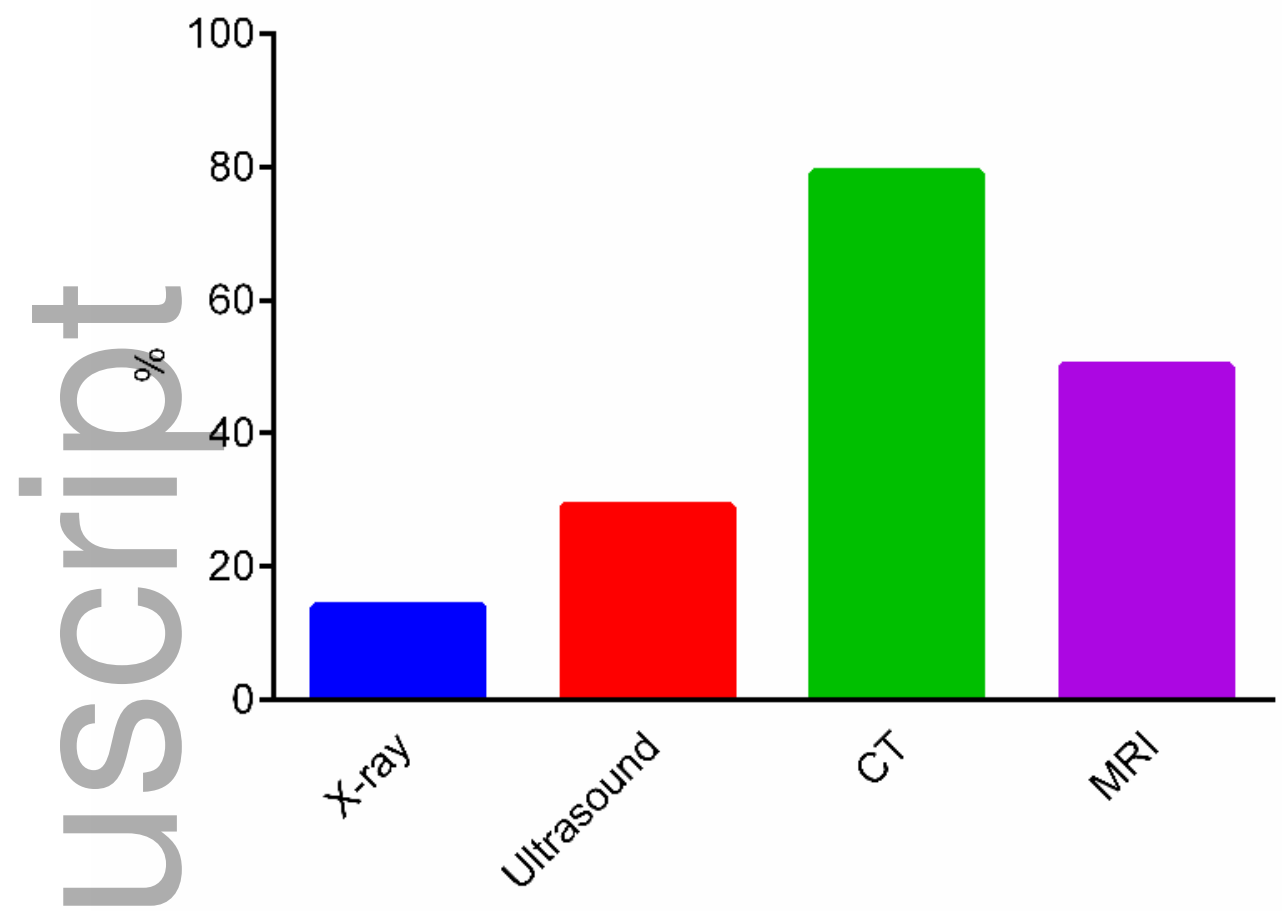

Type of imaging used to plan brachytherapy

ara_12614_f3.tif 


\section{University Library}

\section{- M M N E R VA A gateway to Melbourne's research publications}

Minerva Access is the Institutional Repository of The University of Melbourne

Author/s:

Lim, K;van Dyk, S;Khaw, P;Veera, J;Mileshkin, L;Ohanessian, L;Harrison, M;Vinod, SK

Title:

Patterns of practice survey for brachytherapy for cervix cancer in Australia and New Zealand

Date:

2017-10-01

Citation:

Lim, K., van Dyk, S., Khaw, P., Veera, J., Mileshkin, L., Ohanessian, L., Harrison, M. \& Vinod, S. K. (2017). Patterns of practice survey for brachytherapy for cervix cancer in Australia and New Zealand. JOURNAL OF MEDICAL IMAGING AND RADIATION ONCOLOGY, 61 (5), pp.674-681. https://doi.org/10.1111/1754-9485.12614.

Persistent Link:

http://hdl.handle.net/11343/292865 